\title{
Electrochemical current-sensing atomic force microscopy in conductive solutions
}

\author{
Ilya V Pobelov ${ }^{1, *}$, Miklós Mohos ${ }^{1,2}$, Koji Yoshida ${ }^{1}$, Viliam \\ Kolivoska $^{1}$, Amra Avdic ${ }^{3}$, Alois Lugstein ${ }^{3}$, Emmerich \\ Bertagnolli $^{3}$, Kelly Leonhardt ${ }^{4}$, Guy Denuault ${ }^{4}$, Bernhard \\ Gollas $^{5,6}$ and Thomas Wandlowski ${ }^{1, *}$ \\ ${ }^{1}$ Department of Chemistry and Biochemistry, University of Bern, Freiestrasse 3, \\ CH-3012 Bern, Switzerland. \\ ${ }^{2}$ Institute of Materials and Environmental Chemistry, Research Centre for Natural \\ Sciences, Hungarian Academy of Sciences, 1025 Budapest, Pusztaszeri út 59-67, \\ Budapest, Hungary \\ 3 Solid State Electronics Institute, Vienna University of Technology, Floragasse 7, \\ 1040 Vienna, Austria \\ 4 School of Chemistry, University of Southampton, Highfield, Southampton SO17 \\ 1BJ, United Kingdom \\ ${ }^{5}$ Institute for Chemistry and Technology of Materials, Graz University of \\ Technology, Stremayrgasse 9, 8010 Graz, Austria \\ 6 Competence Centre for Electrochemical Surface Technology, Viktor Kaplan Strasse \\ 2, 2700 Wiener Neustadt, Austria \\ E-mail: ilya.pobelov@iac.unibe.ch, thomas.wandlowski@dcb.unibe.ch
}

\begin{abstract}
Insulated atomic force microscopy probes carrying gold conductive tips were fabricated and employed as bifunctional force and current sensors in electrolyte solutions under electrochemical potential control. The application of the probes for current-sensing imaging, force and current-distance spectroscopy as well as scanning electrochemical microscopy experiments was demonstrated.
\end{abstract}

PACS numbers: 07.79.Lh, 62.25.-g, 82.45.-h, 82.47.Rs, 81.16.-c, 85.85.+j

Submitted to: Nanotechnology 


\section{Introduction}

Current-sensing atomic force microscopy (CSAFM) and spectroscopy have been increasingly employed to characterize mechanical and electrical properties of surfaces, nanostructures and various biosystems. In this approach the force-sensing capability of AFM is combined with the measurement of the current flowing between a bifunctional conductive probe and a conductive sample upon application of a potential difference [1]. The CSAFM technique is employed in three operational modes: (i) Monitoring of the current during AFM imaging allows to distinguish areas with different conductances at the nanoscale [2]. This mode was used to investigate the homogeneity of various organic conductive layers [3], such as polymer electrolyte membranes [4], films employed for photovoltaics [5] and in organic light emitting diodes [6]. (ii) The active force feedback mode is used to create a stable nanojunction, which is characterized by current-voltage spectroscopy. This method was applied to investigate charge transfer characteristics through self-assembled monolayers [7], to address individual nanoparticletagged molecules in an insulating matrix [8], and light-controlled conductance switching [9]. (iii) In current-sensing force spectroscopy experiments both the force acting on the cantilever and the current flowing through a nanoscale junction are measured during approach and retraction of the probe to/from the sample. Applying this technique, the electromechanical properties of gold nanocontacts [10-13] and gold-molecule-gold junctions [11-13] were studied.

Up to now, CSAFM experiments were only carried out in non-conductive environments, such as ultra-high vacuum, air, in a controlled gaseous atmosphere or in non-conductive organic solvents. Important scientific and technological progress is expected if this methodology could be applied in a conductive (electrochemical) environment (figure 1a). However, the preparation of well-insulated, except of the very end, conductive probes (figure 1b) still comprises a major challenge.

Pioneering proof-of-principle experiments were reported by Macpherson et al, who used $\mathrm{Si}_{3} \mathrm{~N}_{4}$ probes metallized with platinum and coated with a thin polymer film [14] or insulated by electrodeposition of electrophoretic paint (EP) [15]. Abbou et al adopted this method to prepare coated gold microwires employed as AFM probes [16]. This group used high voltage pulses of several $\mathrm{kV}$ to expose the very end of the tip.

Although new approaches for the benchtop fabrication of insulated conductive AFM probes are still being communicated [17], more recent research focuses on fabrication procedures based on semiconductor processing techniques, e.g. physical and chemical vapor deposition (PVD/CVD), electron beam lithography (EBL), focused ion beam (FIB) machining, reactive ion etching (RIE), atomic layer deposition (ALD), etc. The latter leads to higher reproducibility and provides opportunities for the batch fabrication of the probes. Kranz et al pioneered the development of AFM probes with an integrated recessed frame [18-21] or ring [22, 23] platinum nanoelectrodes of controlled dimensions around a non-conductive tip for application in combined AFM-scanning electrochemical microscopy (SECM) experiments. These probes were insulated with various coatings, 

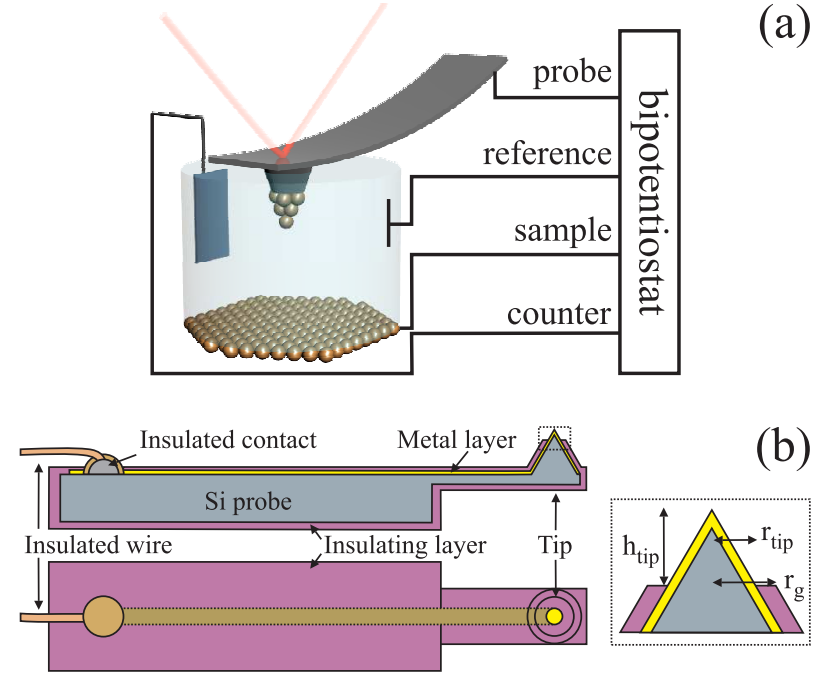

(a)

(b)

Figure 1. (a) Schematics of an electrochemical CSAFM experiment in a 4-electrode configuration. A bipotentiostat controls the potentials of sample $E_{s}$ and probe $E_{p}$ electrodes with respect to a common reference electrode and measures the current flowing through sample $\left(I_{s}\right)$ and probe $\left(I_{p}\right)$. The counter electrode allows to pass the current through the solution. (b) Schematics of a wired and insulated conductive AFM probe. The zoomed frame illustrates the geometric parameters of the tip used to model its electrochemical properties.

such as $\mathrm{Si}_{3} \mathrm{~N}_{4}$ [18, 22], $\mathrm{SiO}_{2}$ [23], $\mathrm{Si}_{3} \mathrm{~N}_{4} / \mathrm{SiO}_{2}$ sandwich layers [19], poly(p-xylylene) (parylene) [20] or plasma-deposited fluorocarbon films [21]. Recently, the group of Kranz reported on diamond-coated tips with integrated boron-doped diamond (BDD) nanoelectrodes [24].

A variety of procedures was employed to fabricate AFM probes with insulated conductive tips (ECCSAFM probes), but only few types were applied for actual CSAFM experiments in an electrochemical environment. ECCSAFM probes were first fabricated by Hirata et al [25], who coated commercial $\mathrm{Si}_{3} \mathrm{~N}_{4}$ probes with gold and insulated them afterwards with a photoresist layer. Akiyama et al [26-28] developed a batch lithographic process to fabricate probes with cone-shaped platinum silicide tips insulated with $\mathrm{Si}_{3} \mathrm{~N}_{4} / \mathrm{SiO}_{2}$. The latter was partially etched to expose the conductive tip. Macpherson and Unwin used EBL to create triangular-shaped gold electrodes at the apex of a $\mathrm{Si}_{3} \mathrm{~N}_{4}$ cantilever as well as contact lines [29]. The whole probes were subsequently insulated with $\mathrm{Si}_{3} \mathrm{~N}_{4}$ and the tips were then exposed by RIE. The insulation of commercial CSAFM probes with parylene and EP was also reported recently [30, 31].

The application of CSAFM in an electrochemical environment is still at a very premature level, despite the progress in probe fabrication. So far all ECCSAFM probes were produced in a small scale and used by the same research group, typically in very basic test experiments. Moreover, establishing and insulation of the electric contact of the probe to the outside world (figure 1) is still poorly described and resolved. To date, the electric contact to the conductive tip was established by a metal clamp [15, 29, 30], 
or a wire $[17-19,28,31]$ attached to the contact pad. The exposed part was subsequently covered with an insulating coating, which was applied manually.

Recently we described the insulation of BDD and Au-covered Si AFM probes with $\mathrm{Si}_{3} \mathrm{~N}_{4}$ [32]. The tip apex was exposed by a combination of FIB and RIE steps. The electrochemical experiments revealed that the $\mathrm{Si}_{3} \mathrm{~N}_{4}$ coating is not pinhole-free and that the conductive tip is partially covered with non-electroactive material, presumably $\mathrm{TiO}_{2}[32,33]$. In this paper we present an improved fabrication procedure, which overcomes these problems: (i) We employed two types of secondary insulations, such as an ALD-fabricated $\mathrm{ZrO}_{2}$ layer or EP, to "post-coat" the $\mathrm{Si}_{3} \mathrm{~N}_{4}$-covered probes. (ii) We removed $\mathrm{TiO}_{2}$ by wet etching. We also describe a reproducible procedure to prepare mount-ready ECCSAFM probes by gluing an insulated wire to the back end of the probe with a conductive epoxy and post-coating the contact with EP (figure 1b). We demonstrate the application of these probes for current-sensing imaging, to measure force/current-distance spectroscopy and SECM-like approach curves [33, 34], as well as for the characterization of electromechanical properties of gold nanocontacts [13]. With this work we aim at providing guidelines for future applications of conductive AFM probes in an electrochemical environment.

\section{Experimental details}

\subsection{Fabrication of probes}

Insulated conductive AFM probes were fabricated using a modified procedure from our previous paper [32]. Commercial Si AFM probes with a pyramidal tip at the end of a $125 \mu \mathrm{m}$ long cantilever (Tap300, BudgetSensors) were sequentially sputter-coated with $5 \mathrm{~nm} \mathrm{Ti}, 120 \mathrm{~nm} \mathrm{Au}$ and $5 \mathrm{~nm}$ Ti layers. The Au layer acts as electrode surface while the Ti layers improve the adhesion of $\mathrm{Au}$ to $\mathrm{Si}$ and $\mathrm{Si}_{3} \mathrm{~N}_{4}$, respectively. The Au-covered probes were insulated by plasma-enhanced chemical vapor deposition of $\mathrm{Si}_{3} \mathrm{~N}_{4}$ at $300^{\circ} \mathrm{C}$ for $50 \mathrm{~min}$, which leads to a nominal film thickness of $600 \mathrm{~nm}$. In addition, a $40 \mathrm{~nm}$-thick $\mathrm{ZrO}_{2}$ insulation layer was deposited on $\mathrm{Si}_{3} \mathrm{~N}_{4}$ using ALD (Savannah 100, Cambridge Nano Tech) according to a previously described procedure [35]. Thereafter, the AFM probes were sputter-coated with a $50 \mathrm{~nm}$ thick sacrificial chromium layer leading to a sandwich structure, as shown in figure 2a. In the next step the $\mathrm{Cr}, \mathrm{ZrO}_{2}$ and $\mathrm{Si}_{3} \mathrm{~N}_{4}$ layers were partly removed at the very apex of the tip with a FIB in a CrossBeam 40EsB dual FIB/SEM system utilizing a beam of $\mathrm{Ga}^{+}$ions at $30 \mathrm{keV}$ and a beam current of $50 \mathrm{pA}$. After this step the conductive tip remains buried underneath the remaining $\approx 150 \mathrm{~nm}$ thick insulation layer. Subsequently, an inductively coupled plasma (ICP)-RIE process was applied using a Plasmalab System 100 (Oxford Instruments). First, a mixture of $\mathrm{SiCl}_{4}$ and $\mathrm{SF}_{6}$ was chosen to remove the Ga-containing top insulation layer. Otherwise it would act as an etch stop during dry etching of $\mathrm{Si}_{3} \mathrm{~N}_{4}$ with $\mathrm{SF}_{6}[36,37]$. Second, $\mathrm{SF}_{6}$ was used to isotropically etch the $\mathrm{Si}_{3} \mathrm{~N}_{4}$ with a high rate, which led to the exposure of the tip. The remaining $\mathrm{Cr}$ protects the rest of the insulation. Height and radius of 


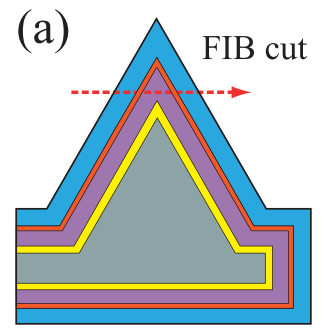

RIE

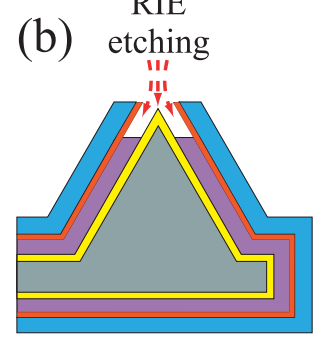

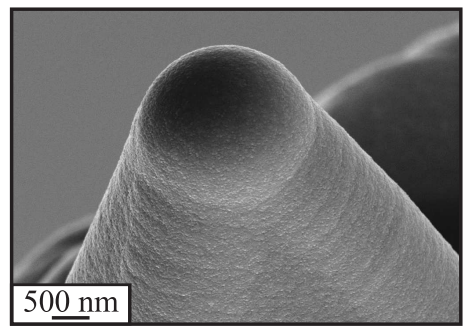

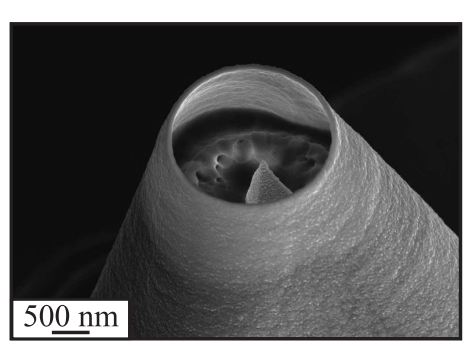

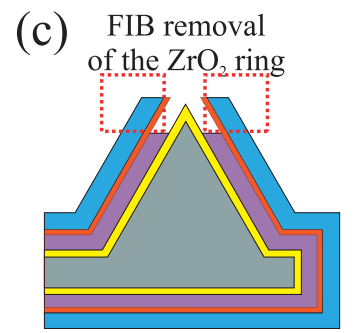

(d)

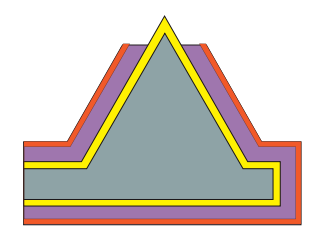

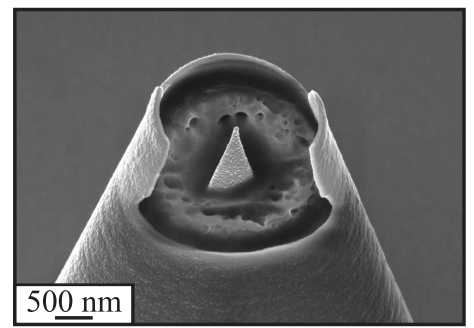

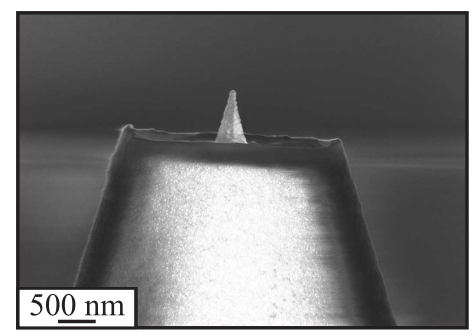

\section{Conventional AFM tip $\square$ Gold layer}

1 Insulation, $\mathrm{Si}_{3} \mathrm{~N}_{4} / \mathrm{ZrO}_{2}$

$\mathrm{Cr}$

Figure 2. Process flow chart of the probe fabrication with the corresponding SEM images (left). (a) AFM tip with a Ti/Au electrode layer, $600 \mathrm{~nm} \mathrm{Si}_{3} \mathrm{~N}_{4}, 40 \mathrm{~nm} \mathrm{ZrO}_{2}$ and a $50 \mathrm{~nm} \mathrm{Cr}$ protection layer. The arrow shows the site for the partial removal of the $\mathrm{Si}_{3} \mathrm{~N}_{4} / \mathrm{ZrO}_{2} / \mathrm{Cr}$ layer by FIB exposing the $\mathrm{Si}_{3} \mathrm{~N}_{4}$ layer for the subsequent etching procedure. (b) Two step ICP-RIE etching of $\mathrm{Si}_{3} \mathrm{~N}_{4}$ exposing the tip and forming a $\mathrm{ZrO}_{2} / \mathrm{Cr}$ ring around the tip. (c) Removal of the ring by a FIB cut. (d) Fully featured probe after removal of the Cr layer by wet etching.

the exposed tip depend on the etching time, which is well controllable. The $\mathrm{ZrO}_{2}$ layer remained intact after this procedure, creating a $\mathrm{ZrO}_{2} / \mathrm{Cr}$ ring around the tip (figure $2 \mathrm{~b}$ ). At this stage, a second FIB step was used to remove this ring. The probe was placed in the FIB system with the ring positioned perpendicular to the beam, and then the side portions of the ring were removed (figure 2c). Subsequently, the probe was turned $90^{\circ}$ and the rest of the ring was removed by FIB cutting. After the removal of the ring, the rest of the Cr coating was dissolved by wet etching employing Microposit chrome etch 18 (figure $2 \mathrm{~d}$ ).

The Ti adhesion layer, which was originally located between the $\mathrm{Au}$ and the $\mathrm{Si}_{3} \mathrm{~N}_{4}$ layers, was partially removed from the tip and partially oxidized during etching with $\mathrm{SiCl}_{4} / \mathrm{SF}_{6}$. The residual $\mathrm{TiO}_{2}$ layer, which covered the tip and therefore reduced the electro-active area, was removed by immersing the $\mathrm{AFM}$ probe for $1 \mathrm{~min}$ at $60^{\circ} \mathrm{C}$ into an aqueous solution of $60 \mathrm{mM} \mathrm{Na}_{2} \mathrm{EDTA}+370 \mathrm{mM} \mathrm{NH} 4 \mathrm{OH}+1100 \mathrm{mM} \mathrm{H}_{2} \mathrm{O}_{2}$.

The stability of the $\mathrm{Al}_{2} \mathrm{O}_{3}$ and $\mathrm{ZrO}_{2}$ layers, which were fabricated by ALD, was tested using probes of a simpler design. The metal oxides were deposited directly onto the Au layer. 


\subsection{Chemicals and materials}

Milli-Q water (Millipore, resistivity $18.2 \mathrm{M} \Omega \cdot \mathrm{cm}, 2 \mathrm{ppb}$ total organic carbon) was used for cleaning and for the preparation of the solutions. The solutions used in the electrochemical and AFM experiments were prepared from sulfuric acid $\mathrm{H}_{2} \mathrm{SO}_{4}(96 \%$, suprapure, Merck), potassium nitrate $\mathrm{KNO}_{3}(\geq 98 \%$, Fluka) and ferrocenemethanol FcMeOH (97\%, Aldrich). Argon (99.999\%, Alphagaz) was used to dry the probes and to deoxygenate the electrolytes before and during the electrochemical experiments.

\subsection{Wiring of the probes}

All probes employed in this work were connected to the measurement circuit via a thin copper wire, which was insulated with polyurethane (BLOCK CUL 200/ø0.15 mm, $\approx 5 \mathrm{~cm}$ long). First, the contact area was created on the working surface of the probe on the side opposite to the cantilever (figure 1b) by slightly scratching the insulation layer. The end of the wire was covered with a small amount of tin. Then, a small droplet of conductive two-component epoxy (Circuit Works 2400) was picked up by the wire and was gently positioned on the contact area. The probe was then cured in an oven for one hour at $110^{\circ} \mathrm{C}$. This procedure creates a mechanically stable and well-conducting contact between the inner conductive layer of the probe and the insulated wire. For electrochemical experiments, such as cyclic voltammetry (CV) and EP deposition, the free end of the insulated wire was soldered to a thicker copper wire $(\varnothing 0.5 \mathrm{~mm}, 3-4 \mathrm{~cm}$ long), which was inserted into a lab-made capillary-type electrode holder.

\subsection{Insulating probes with electrophoretic paint}

The electric contact between the probe and the connecting wire was insulated by deposition of an EP (Clearclad HSR, Clearclad Inc.). The probe was cleaned by immersion into Milli-Q water, gently dried in an Ar stream, mounted in the electrode holder and immersed into the homogenized EP in a backward manner, with the cantilever and the front part of the chip being kept above the surface of the liquid. This prevented the conductive tip from being coated. The deposition process was carried out in a two-electrode configuration with the AFM probe and a $\mathrm{Pt}$ wire acting as cathode and anode, respectively, by applying a potential difference of $15 \mathrm{~V}$ DC for 10 min. Subsequently, the probe was cured in an oven at $80^{\circ} \mathrm{C}$ for 8 hours. The coating-curing process was repeated three times. Finally, the probe was tempered for 1 hour at $160^{\circ} \mathrm{C}$. These conditions enhanced the polymerization and increased the adhesion of the polymer layer.

\subsection{Electrochemical characterization}

The electrochemical characterization of the probes was carried out by cyclic voltammetry in a 3-electrode glass cell using a lab-built potentiostat capable of measuring subpicoampere currents [38]. All glassware used was cooked in boiling nitric acid (25\% 
in water v:v) and extensively rinsed with Milli-Q water. A saturated calomel electrode ( $\mathrm{SCE}$ ) and a $\mathrm{Pt}$ wire served as reference (RE) and counter (CE) electrodes, respectively. Ar was used to purge the solution prior to and was directed above it during the measurements. The step-wise immersion of the AFM probe into the electrolyte solution was performed by a micrometer screw attached to the electrode holder with the AFM probe mounted on it.

\subsection{Electrochemical current-sensing AFM experiments}

The electrochemical AFM experiments were carried out in contact mode using a modified PicoSPM 5500 (Agilent) setup equipped with a $10 \mu \mathrm{m}$ multi-mode AFM scanner with a contact mode nose cone. PicoView 1.12 was employed as controlling software. The parts of the AFM scanner, which were in contact with the solution during the measurements, were rinsed with isopropanol and dried in an Ar stream. The wired AFM probe was cleaned by immersion into Milli-Q water and gently dried in an $\mathrm{Ar}$ stream prior to mounting into the AFM scanner. The probe was held with a metal clamp. A build-in bipotentiostat (Agilent) was used to control the potentials of probe and sample. The probe potential was kept at ground during all measurements. The insulated wire attached to the probe was connected to a lab-built dual-channel linear amplifier. A lab-built electronics measured the current flowing through the probe and controlled the probe movement in the current-sensing force spectroscopy experiments [13]. Either the low or the high current output of a dual-channel amplifier was fed back into the PicoPlus controller as an Aux/CSAFM signal for data acquisition during the electrochemical characterization of the probe (figure 4) or during the current-mapping and the force/current-distance spectroscopic experiments (figures 5, 6).

Two samples were employed in the AFM experiments: (1) A nanostructured $\mathrm{Si}$ wafer was used in the current-mapping experiments. Its surface was covered with a 1 $\mu$ m-thin insulating $\mathrm{SiO}_{2}$ layer onto which $200 \mathrm{~nm}$-thick gold lines of variable width were deposited using optical lithography. (2) The second sample was a lab-built Claviliertype gold bead electrode supported by a gold sheet with the (111) facet exposed to the probe. These electrodes were employed in force and current measurements upon forming and pulling gold nanocontacts. The samples were cleaned as follows prior to the measurements. Sample 1 was immersed into chloroform (98\%, Arcos Organics) and ethanol (94\%, Dr Grogg Chemie AG, Basel), and dried in an Ar stream. Sample 2 was electropolished by oxidizing its surface at $5 \mathrm{~V}$ vs a gold $\mathrm{CE}$ in $1 \mathrm{M} \mathrm{H}_{2} \mathrm{SO}_{4}$ for 5-10 s. The gold oxide was then removed by immersing the sample without potential control into $1 \mathrm{M} \mathrm{HCl}$ solution for $1 \mathrm{~min}$. This cycle was repeated three times. The electrode was rinsed with copious amounts of Milli-Q water after each step. Finally, the electrode was flame annealed in a butane flame and cooled down in an Ar stream.

The AFM samples were mounted on a lab-built sample plate. An O-ring (16 $\mathrm{mm} \times 2 \mathrm{~mm}$ for sample 1 or $6.07 \mathrm{~mm} \times 1.78 \mathrm{~mm}$ for sample 2, Kalrez 4079, DuPont) and the corresponding Kel-F liquid cell were placed onto the sample and fixed by a 
metal holder. The cell was then immediately filled with electrolyte. The O-rings and liquid cells were cleaned in caroic acid (3:1 v:v conc. $\mathrm{H}_{2} \mathrm{SO}_{4}$ and $30 \% \mathrm{H}_{2} \mathrm{O}_{2}$, CAUTION: extremely strong oxidant), rinsed with copious amounts of Milli-Q water and dried in an Ar stream before mounting. Two Pt wires $(99.999 \%, \varnothing 0.5 \mathrm{~mm}$, Goodfellow) served as RE and CE. All potentials given in this paper refer to the SCE scale, and were recalculated from the individual experiments accordingly. The AFM experiments were carried out in the presence of ambient oxygen.

\section{Results and discussion}

\subsection{Fabrication and electrochemical characterization of the insulated conductive AFM probes}

In our previous publication we characterized the electrochemical properties of the $\mathrm{Si}_{3} \mathrm{~N}_{4^{-}}$ insulated probes by immersing their front parts into the electrolyte solution. Based on cyclic voltammetry, we demonstrated that these probes have well-defined conductive tips [32]. Later we carried out a detailed investigation of the insulation (leakage and stability) of the entire chip by a step-wise immersion of the probe into the solution (figure 3 ). This procedure allows to quickly detect defects within the insulation at the probe surface, and to estimate the corresponding leakage currents (cf figure 1). We found that the $\mathrm{Si}_{3} \mathrm{~N}_{4}$-insulated probes display a continuous increase of the electrochemical current upon immersion (SI, section A) indicating an increase of the electroactive area. Thus, these probes have many pinholes in the insulating layer, which prevent their application in electrochemical AFM experiments.

Aiming to solve the problem of leakage currents, we added a second insulation layer. In a first attempt, we modified our fabrication procedure by insulating the goldcoated probes with a metal oxide layer $\left(\mathrm{ZrO}_{2}\right.$ and $\left.\mathrm{Al}_{2} \mathrm{O}_{3}\right)$ using the ALD technique (paragraph 2.1). This approach is known to produce uniform and compact layers [35]. Prior to the fabrication of probes with exposed tips, we fabricated a series of gold-coated probes completely insulated with a thin layer of $\mathrm{ZrO}_{2}$ or $\mathrm{Al}_{2} \mathrm{O}_{3}$, and investigated their stability in $0.1 \mathrm{M} \mathrm{KNO}_{3}$ and $0.1 \mathrm{M} \mathrm{H}_{2} \mathrm{SO}_{4}$, both representing typical environments of electrochemical experiments (SI, section B). We found that the $\mathrm{Al}_{2} \mathrm{O}_{3}$ insulation is only stable in neutral electrolytes, such as $0.1 \mathrm{M} \mathrm{KNO}_{3}$, while $\mathrm{ZrO}_{2}$ is stable within a broad range of electrode potentials in neutral as well as in acidic environment.

Further, we fabricated probes with exposed gold tips insulated by combining $\mathrm{Si}_{3} \mathrm{~N}_{4}$ and $\mathrm{ZrO}_{2}$ coatings (figure 2), and characterized them electrochemically. Cyclic voltammograms of the insulated and wired probes were obtained upon its gradual immersion into an aqueous solution of $0.1 \mathrm{M} \mathrm{KNO}_{3}$ containing $1 \mathrm{mM}$ FcMeOH as reactant. Figure 3a demonstrates that the same current response was measured with either only the cantilever or with more than half of the chip being immersed in the electrolyte (positions 1 and 2). These experiments revealed a well-defined sigmoidal current response with shape and height being rather independent of the potential sweep 


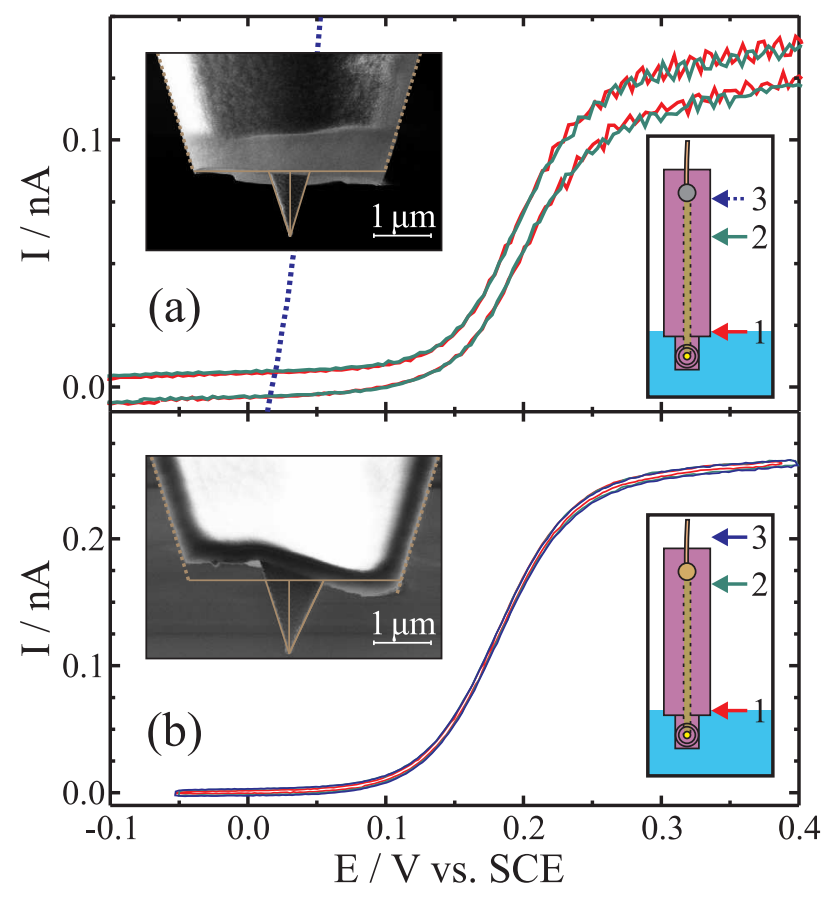

Figure 3. Cyclic voltammograms of gold-coated CSAFM probes insulated with $\mathrm{Si}_{3} \mathrm{~N}_{4} / \mathrm{ZrO}_{2}$ (a) or $\mathrm{Si}_{3} \mathrm{~N}_{4} / \mathrm{EP}$ (b) measured upon their gradual immersion in $0.1 \mathrm{M}$ $\mathrm{KNO}_{3}+1 \mathrm{mM} \mathrm{FcMeOH}$. The potential sweep rate was $0.05 \mathrm{~V} \cdot \mathrm{s}^{-1}$. The dotted curve in (a) indicates the current measured when the contact entered the solution at position 3. The left insets show the SEM micrographs representing the geometries of the tips used. The solid lines illustrate an approximation of the tip geometries as used in simulations. The right insets show the positions of the probes with respect to the surface of the electrolyte upon immersion of the probe.

rate. The electrochemical response corresponds to the oxidation of FcMeOH on a welldefined nanoelectrode with the limiting current controlled by the diffusion of FcMeOH. A sharp increase of the current was observed upon further immersion of the probe up to the position of the contact to an external wire (position 3), as indicated by the dotted line in figure $3 \mathrm{a}$.

In a second double-coating procedure, we combined the $\mathrm{Si}_{3} \mathrm{~N}_{4}$ insulation of the probes [32] with an electrochemical post-coating step. After wiring of a $\mathrm{Si}_{3} \mathrm{~N}_{4}$-coated probe, EP was deposited on the whole probe surface (except of the tip) and on the electric contact of the probe (see paragraph 2.4). We avoided in this way a separate step for the insulation of the latter. Figure $3 b$ shows typical cyclic voltammograms, as recorded during the step-wise immersion of a $\mathrm{Si}_{3} \mathrm{~N}_{4}$-insulated $\mathrm{Au}$ probe freshly-coated with EP into an electrolyte containing $0.1 \mathrm{M} \mathrm{KNO}_{3}$ and $1 \mathrm{mM} \mathrm{FcMeOH}$. The sigmoidal voltammogram was independent on the position of the probe, which reflects the absence of insulation defects on the surface of the probe as well as on the electric contact.

As the ability to predict the expected electrochemical current is very important in experiments with nanoelectrodes, we used our electrochemical results to highlight the correlation between tip current and tip geometry. We compared the experimental 
Table 1. Tip geometries, calculated [33] and experimentally measured diffusionlimited currents of $\mathrm{FcMeOH}$ oxidation for the probes presented in figures $3 \mathrm{a}$ and $3 \mathrm{~b}$.

\begin{tabular}{lllllll}
\hline & $\begin{array}{l}r_{\text {tip }} \\
(\mathrm{nm})\end{array}$ & $\begin{array}{l}h_{\text {tip }} \\
(\mathrm{nm})\end{array}$ & $\begin{array}{l}r_{g} \\
(\mathrm{~nm})\end{array}$ & $\begin{array}{l}\text { Area } \\
\left(\mu \mathrm{m}^{2}\right)\end{array}$ & $\begin{array}{l}I_{d, \text { calc }} \\
(\mathrm{nA})\end{array}$ & $\begin{array}{l}I_{d, \exp } \\
\mathrm{nA}\end{array}$ \\
\hline $3 \mathrm{a}$ & 360 & 1145 & 1680 & 1.36 & 0.25 & 0.13 \\
$3 \mathrm{~b}$ & 510 & 1290 & 1860 & 2.22 & 0.31 & 0.26 \\
\hline
\end{tabular}

diffusion-limited current of the FcMeOH oxidation $I_{d}$, as measured by the two insulated probes (figure 3), with the theoretical values determined according to recently derived analytical expressions [33]. We first approximated the shape of the probes as depicted in the SEM micrographs by three parameters: the height and the radius of the electroactive cone, $h_{\text {tip }}$ and $r_{\text {tip }}$, and the radius of the insulation $r_{g}$ (cf figure 1b). Employing these parameters, we calculated $I_{d}$ for the oxidation of $1 \mathrm{mM} \mathrm{FcMeOH}$ (diffusion coefficient $D=7.8 \times 10^{-6} \mathrm{~cm}^{2} \cdot \mathrm{s}^{-1}$ [39]) as described in SI, section C and [33] (table 1). These results are rather close to the experimental values. The difference between $I_{d, \text { calc }}$ and $I_{d, \exp }$ could be related to unavoidable errors upon estimation of the geometric parameters, as well as to deviations of the tip shapes from an ideal one [33]. For comparison, $I_{d, \text { calc }} \approx 4 \times I_{d \text {,exp }}$ was previously obtained for another Au-coated tip [32]. We believe that the removal of titanium oxide from the gold tip, as implemented in our new experiments, comprises the critical step to obtain a better agreement between calculated and experimental currents.

\subsection{Current-sensing AFM experiments in an electrochemical environment}

We used those insulated probes, which showed a low leakage current during the electrochemical characterization, in subsequent ECCSAFM experiments (see paragraph 2.6 for details). The first series of experiments was carried out with a sample consisting of conductive gold lines on an insulating $\mathrm{SiO}_{2}$ surface in $0.05 \mathrm{M} \mathrm{H}_{2} \mathrm{SO}_{4}+0.05 \mathrm{M}$ $\mathrm{KNO}_{3}+0.5 \mathrm{mM} \mathrm{FcMeOH}$ and the probe insulated with $\mathrm{Si}_{3} \mathrm{~N}_{4} / \mathrm{EP}$ as presented in figure 3b. Figure 4 illustrates $\mathrm{CVs}$ of the sample and of the AFM probe as recorded in the AFM cell. Both CVs show the characteristic responses of FcMeOH oxidation controlled by planar diffusion (sample macroelectrode) or hemispherical diffusion (tip nanoelectrode). The overall shape of the redox response of the probe as well as the capacitance response in the double layer region are comparable with the data recorded in the electrochemical cell in the absence of oxygen (figure 3b). However, the height of the oxidation wave is 3 to 4 times larger despite the lower concentration of $\mathrm{FcMeOH}$. This effect is attributed to a degradation of the EP-coating as well as to its damage during handling, in particular during the mounting of the probe into the AFM scanner. However, already at this stage the leakage current of the probe was sufficiently small to carry out proof-of-principle experiments, which will be presented next. We note that the negative probe current was measured in the AFM cell at potentials $E<0.15 \mathrm{~V}$. This current is attributed to the reduction of oxygen, which, unlike in experiments carried out 
in an electrochemical cell (paragraph 3.1), is dissolved in the electrolyte under ambient conditions in the AFM setup employed in the current study.

The gold lines on the $\mathrm{SiO}_{2}$ surface were imaged by contact-mode AFM in electrolyte under potential control with a resolution below $100 \mathrm{~nm}$ (figure 5a). The simultaneously mapped current, which flows through the junction (figure 5b), allows to distinguish conductive and insulating parts of the structured Si wafer. This approach helps to discern between domains with different electric properties on more complex samples. The conductance of different domains can be quantified in a current histogram (figure 5c). We observed two distinct features. While the majority of data points in

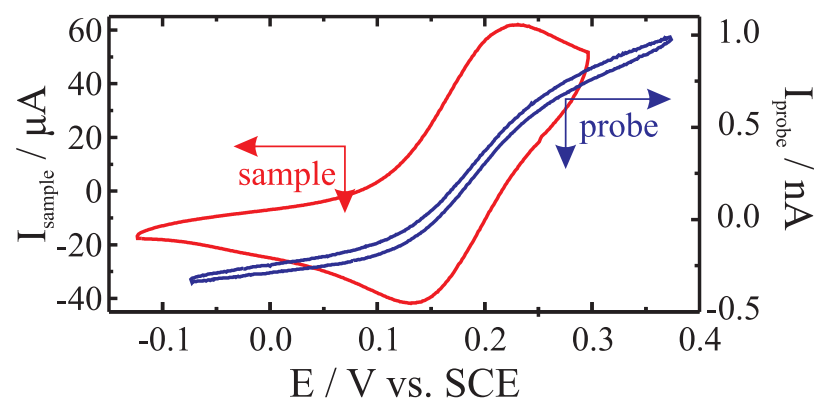

Figure 4. Cyclic voltammograms of the sample (scale on the left) and of the AFM probe (scale on the right) in $0.05 \mathrm{M} \mathrm{H}_{2} \mathrm{SO}_{4}+0.05 \mathrm{M} \mathrm{KNO}_{3}+0.5 \mathrm{mM} \mathrm{FcMeOH}$ as measured in the AFM cell. The potential sweep rate is $0.05 \mathrm{~V} \cdot \mathrm{s}^{-1}$.

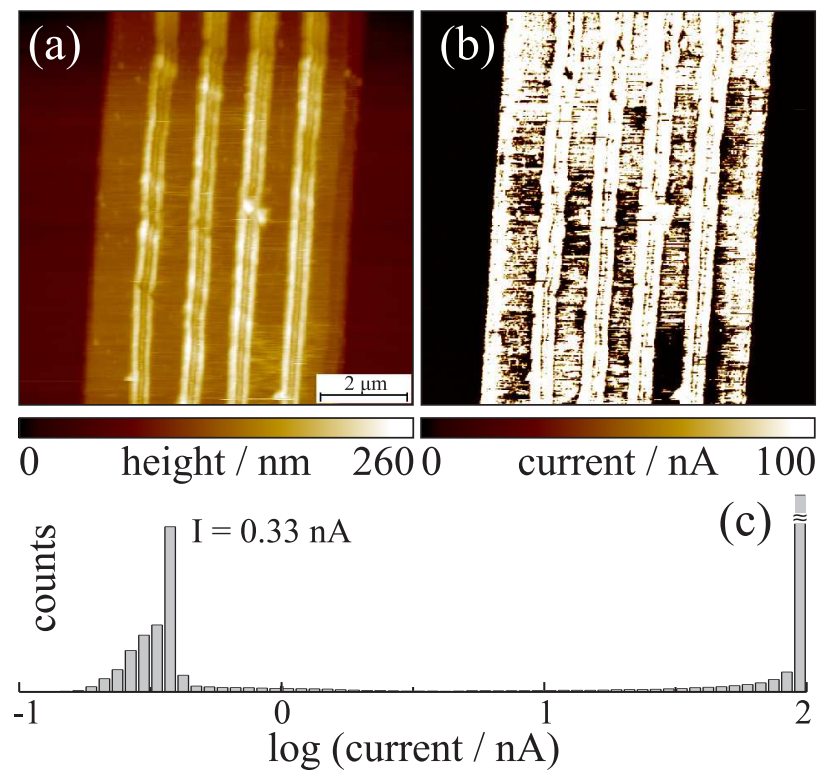

Figure 5. Simultaneous mapping of topography (a) and current (b) upon AFM imaging in $0.05 \mathrm{M} \mathrm{H}_{2} \mathrm{SO}_{4}+0.05 \mathrm{M} \mathrm{KNO}_{3}+0.5 \mathrm{mM} \mathrm{FcMeOH}, E_{s}=0 \mathrm{~V}, E_{p}=-0.1 \mathrm{~V}$ vs SCE. (c) Histogram of the current values in (b) showing an electrochemical current $I \approx 0.33 \mathrm{nA}$ and a saturation current $I \approx 100 \mathrm{nA}$ upon contacting the conductive lines. 

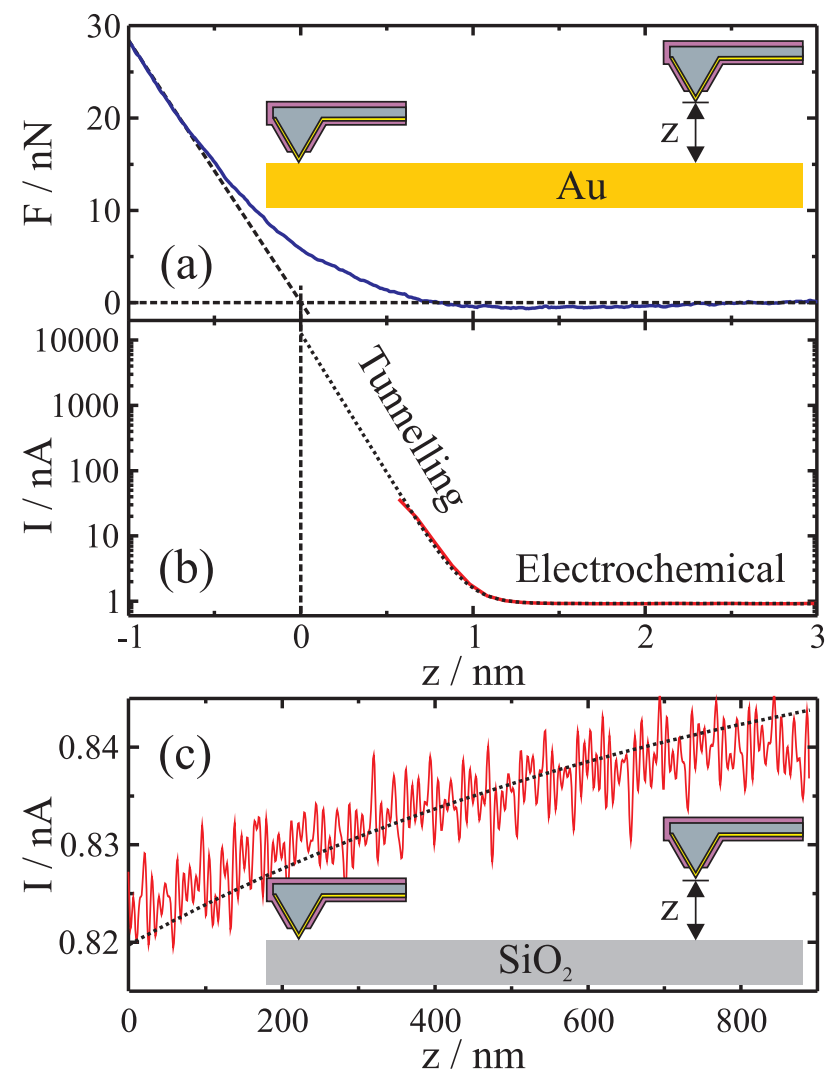

Figure 6. Curves measured upon the approach of the probe towards the surface in $0.05 \mathrm{M} \mathrm{H}_{2} \mathrm{SO}_{4}+0.05 \mathrm{M} \mathrm{KNO}_{3}+0.5 \mathrm{mM} \mathrm{FcMeOH}, E_{s}=0 \mathrm{~V}, E_{p}=0.3 \mathrm{~V}$ vs SCE , probe moving rate $100 \mathrm{~nm} \cdot \mathrm{s}^{-1}$ (solid lines). (a) Force and (b) current on a conducting gold line. The dashed lines in (a) demonstrate the procedure used to determine the position of the probe-sample contact. The dotted curve in (b) corresponds to the sum of simulated $\mathrm{FcMeOH}$ oxidation current, tunnelling current and a constant offset current of $0.68 \mathrm{nA}$. (c) Current measured upon probe approach to the insulating $\mathrm{SiO}_{2}$ surface. The dotted curve corresponds to the sum of simulated FcMeOH oxidation current and a constant offset current of $0.71 \mathrm{nA}$.

the last bin corresponds to the saturation current $I \approx 100 \mathrm{nA}$ flowing between the probe and the sample upon their contact, the smaller peak centred at $I \approx 0.33 \mathrm{nA}$ is attributed to the current measured when the probe is in contact with the nonconductive areas. The current measured at the probe potential $E_{p}=-0.1 \mathrm{~V}$ is due to the oxygen reduction and is approximately 1-2 orders of magnitude higher than the typical electrochemical currents obtained with well insulated tips in electrochemical scanning tunnelling microscopy [40] and, according to our estimations, less than 1 order of magnitude higher than the background current typically measured in AFM-SECM experiments.

The surface features were characterized by measuring simultaneously the force $F$ acting on the cantilever and the current $I$ flowing through the junction as a function of probe-sample distance $z$ upon approach and withdrawal of the AFM probe to the conductive and to the non-conductive areas. This technique is known as force-distance 
or current-distance spectroscopy in atomic force and scanning tunnelling microscopy configurations, respectively. As probe and sample potentials were held at values corresponding to the diffusion-limited oxidation of $\mathrm{FcMeOH}$ and its regeneration upon reduction of $\mathrm{FcMeOH}^{+}$, our setting is also equivalent to a typical SECM experiment. The experiments were complemented by modelling the current of the diffusion-limited oxidation of $\mathrm{FcMeOH}$ at the probe/electrolyte interface upon the approach of the probe to the conductive and to the non-conductive surface (for details see SI, section C).

Figures $6 \mathrm{a}$ and $6 \mathrm{~b}$ display the typical evolution of force and current during the approach of the probe to a conductive gold line. Here, the scale corresponds to the absolute probe-sample distance. The probe-sample contact $(z=0)$ was determined as the point where the initially resting cantilever $(F=0)$ starts to push against the sample ( $F$ increases) by extrapolation of the linear $F-z$ dependence to zero force (dotted lines in figure $6 \mathrm{a}$ ). A constant current $I_{p} \approx 0.9 \mathrm{nA}$ corresponding to the diffusion-controlled oxidation of FcMeOH (cf figure 4) was measured when the probe was sufficiently far from the surface. It increased sharply when the probe-sample distance was below $1 \mathrm{~nm}$. Two mechanisms could be responsible for the observed current increase: regeneration of $\mathrm{FcMeOH}^{+}$(positive feedback in SECM) or electron tunnelling between the probe and the sample. The transition from the first mechanism to the second one upon the approach of the probe to the surface was also observed experimentally [41]. However, the corresponding simulated SECM response (SI, figure S8) demonstrated a slow current increase over a distance of several $\mu \mathrm{m}$ from the surface reaching $\Delta I \approx 0.1 \mathrm{nA}$ at most. The characteristic exponential current-distance dependence at $z<1 \mathrm{~nm}$ in figure $6 \mathrm{~b}$ is therefore rather attributed to electron tunnelling. The current-distance curve was reproduced as a sum of simulated $\mathrm{FcMeOH}$ oxidation current, constant offset of $0.68 \mathrm{nA}$, and a tunnelling contribution with an estimated barrier height of $\approx 0.95 \mathrm{eV}$. This value is slightly lower than 1.0 to $1.5 \mathrm{eV}$, obtained for typical tunnelling experiments in aqueous electrolytes with $\mathrm{Pt} / \mathrm{Ir}$ and $\mathrm{Au}$ tips in the absence of redox-active species $[42,43]$. The extrapolation of the tunnelling current to $z=0$ results in a contact conductance of $42.6 \mu \mathrm{S}$, which is slightly smaller than the conductance of a single-atom gold-gold contact (see below).

Approaching of the probe to a non-conductive surface results in a slight decrease of the current from $\approx 0.84 \mathrm{nA}$ to $\approx 0.82 \mathrm{nA}$ over a distance of $\approx 1 \mu \mathrm{m}$ (figure $6 \mathrm{c}$ ), while the simulated approach curve (SI, figure S8) displays a current of $\approx 0.15 \mathrm{nA}$ when the probe is located sufficiently far away from the surface. However, the decrease of the simulated current due to the screening of the FcMeOH diffusion to the tip, as described by a negative feedback in SECM, corresponds well to the variation of the experimental current. Thus, we were able to reproduce the experimental response as a sum of simulated FcMeOH oxidation current and a constant offset of $0.71 \mathrm{nA}$. We attribute the offset current observed in both types of current-distance measurements (figure 6b,c) to a leakage current. Possible origins of the latter were discussed above.

Finally, we used an experimental configuration, which we already described before [13], to demonstrate simultaneous current and force measurements upon pulling of gold- 


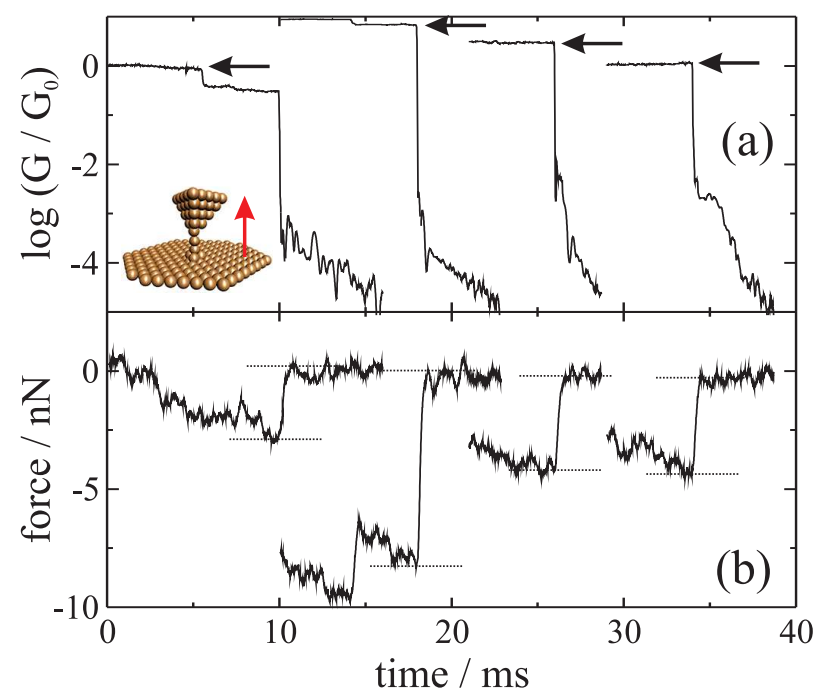

Figure 7. Examples of traces showing the evolution of the junction conductance (a) and of the force (b) upon stretching of Au nanocontacts in $0.1 \mathrm{M} \mathrm{H}_{2} \mathrm{SO}_{4}$, pulling rate $100 \mathrm{~nm} \cdot \mathrm{s}^{-1}, E_{s}=-0.15 \mathrm{~V}, E_{p}=-0.28 \mathrm{~V}$ vs SCE.

gold nanocontacts. These contacts were created between an AFM probe and a gold bead crystal in $0.1 \mathrm{M} \mathrm{H}_{2} \mathrm{SO}_{4}$ (figure 7 ). The formation of gold-gold nanocontacts was identified by a well-defined conductance $G \approx N \cdot G_{0}$, where $G_{0}=77.5 \mu \mathrm{S}$ represents the quantum of conductance and $N$ is the number of quantum conductance channels in the narrowest part of the nanocontact (see arrows in figure 7). After breaking of an atomic contact, the junction conductance rapidly drops by a few orders of magnitude followed by an exponential decrease in conductance. This decay is attributed to tunnelling through the solution. At the same time, the force acting on the cantilever suddenly increases by a few $\mathrm{nN}$. The force jump is attributed to the force required to break the last stable atomic contact [10-13]. This force is known to be $\approx 1.5 \mathrm{nN}$ for a singleatom gold contact under non-reactive conditions. However, it appears to be somewhat higher under electrochemical conditions. The detailed analysis and the discussion of the potential-dependence of the electromechanical properties of gold nanocontacts in an electrochemical environment are the subject of a forthcoming publication.

\section{Conclusions}

In this paper we present (1) an improved technology for the fabrication of atomic force microscopy probes with insulated conductive tips; (2) a procedure to create a reliable contact between the internal conductive structure of the probe and an external current-measuring circuit; (3) an approach to apply a secondary insulation to the electric contact and to the surface of the probe by electrodeposition of an electrophoretic paint; (4) a protocol for the characterization of probe insulation with a specific focus on its stability in a reactive environment; (5) prototypical electrochemical scanning probe 
microscopy/spectroscopy experiments under potential control employing the fabricated probes as bifunctional force/current sensors; as well as (6) the analysis strategies for the latter experiments.

Compared to the fabrication process reported previously [32], we improved the insulation of the conductive probes by combination of plasma-enhanced chemical vapor deposition of $\mathrm{Si}_{3} \mathrm{~N}_{4}$ with (1) atomic layer deposition of $\mathrm{ZrO}_{2}$ or, alternatively, (2) electrochemically-deposited electrophoretic paint. Both approaches lead to wellinsulated probes, except of the conductive tips exposed to the electrolyte. While the ALD strategy still requires insulation of the electric connection to the external circuit, electrophoretic deposition allows to obtain a defect-free insulation of the whole probe and of the electric contact. Particularly promising results were obtained with probes coated and cured shortly before the experiments. The stability of the insulation layers was confirmed by experiments with wired probes, that were characterized by cyclic voltammetry in $0.1 \mathrm{M} \mathrm{KNO}_{3}, 0.1 \mathrm{M} \mathrm{H}_{2} \mathrm{SO}_{4}$ or $0.1 \mathrm{M} \mathrm{KNO}_{3}+1 \mathrm{mM} \mathrm{FcMeOH}$. We found that the maximum diffusion-limited current of $\mathrm{FcMeOH}$ oxidation at the tip/electrolyte interface agrees almost quantitatively with simulated currents obtained according to a previously reported procedure [33].

The wired and well-insulated probes were further employed in prototype ECCSAFM experiments. The electroactive area of the probes, as determined in the ECCSAFM experiments, was found to be 7 to 8 times higher than that extracted from their characterization in a standard electrochemical cell. This observation is attributed to microscratches imposed on the probe during its handling. The latter still needs technical improvements. However, the fabricated insulated probes already allowed proofof-principle experiments, which illustrate the feasibility of our approach.

In a first current-sensing AFM experiment we imaged conductive gold lines on an insulating $\mathrm{SiO}_{2}$ surface. We convincingly demonstrated that mapping of surface structures with different conductances is possible in electrolyte. In a second experiment, we measured the force acting on the cantilever as well as the current flowing through the junction during the approach of the probe to the conductive and to the non-conductive surfaces. With the potentials of the sample and of the probe corresponding to the stability range of the reduced and of the oxidized form of $\mathrm{FcMeOH}$, this experiment is equivalent to force- or current-distance scanning probe spectroscopy as well as to approach curves in scanning electrochemical microscopy (SECM). We found that the current increase upon approaching the probe to a conductive area of the sample (at distances $z<1 \mathrm{~nm}$ ) represents electron tunnelling between the two electrodes. The decrease of the current upon the approach of the probe to an insulating area of the sample was interpreted to be due to the hindering of the $\mathrm{FcMeOH}$ diffusion to the probe, as confirmed by complementary simulations [34]. Finally, we demonstrated, for the first time in an electrochemical environment, simultaneous measurements of interaction forces and junction conductances upon breaking of metal nanocontacts. These experiments open the way towards the exploration of structure and reactivity of nanojunctions in an electrochemical environment. 
We believe that our work will provide guidelines for the fabrication and characterization of insulated CSAFM probes and inspire advanced CSAFM experiments in a wide range of electrochemical applications.

\section{Acknowledgments}

This work was supported by the University of Bern, the Swiss National Science Foundation (200020-144471, 200021-124643, NFP 62, Sinergia CRSII2 126969/1), the Swiss Commission for Technology and Innovation (Project 13696.1), the COMET program of the Austrian Research Advancement Agency (Österreichische Forschungförderungsgesellschaft FFG) and the government of Lower Austria. GD acknowledges the Hanse-Wissenschaftskolleg for the award of a fellowship.

\section{References}

[1] Jeong Y. Park, Sabine Maier, Bas Hendriksen, and Miquel Salmeron. Sensing current and forces with SPM. Mater. Today, 13:38-45, 2010.

[2] F. Houzé, R. Meyer, O. Schneegans, and L. Boyer. Imaging the local electrical properties of metal surfaces by atomic force microscopy with conducting probes. Appl. Phys. Lett., 69:1975-1977, 1996.

[3] Yabing Qi. Investigation of organic films by atomic force microscopy: structural, nanotribological and electrical properties. Surf. Sci. Rep, 66:379-393, 2011.

[4] Elena Aleksandrova, Renate Hiesgen, K. Andreas Friedrich, and Emil Roduner. Electrochemical atomic force microscopy study of proton conductivity in a nafion membrane. Phys. Chem. Chem. Phys., 9:2735-2743, 2007.

[5] David C. Coffey, Obadiah G. Reid, Deanna B. Rodovsky, Glenn P. Bartholomew, and David S. Ginger. Mapping local photocurrents in polymer/fullerene solar cells with photoconductive atomic force microscopy. Nano Lett, 7:738-744, 2007.

[6] L. S. C. Pingree, M. C. Hersam, M. M. Kern, B. J. Scott, and T. J. Marks. Spatially-resolved electroluminescence of operating organic light-emitting diodes using conductive atomic force microscopy. Appl. Phys. Lett., 85:344-346, 2004.

[7] David J. Wold and C. Daniel Frisbie. Fabrication and characterization of metalmolecule-metal junctions by conducting probe atomic force microscopy. J. Am. Chem. Soc., 123:5549-5556, 2001.

[8] X. D. Cui, A. Primak, X. Zarate, J. Tomfohr, O. F. Sankey, A. L. Moore, T. A. Moore, D. Gust, G. Harris, and S. M. Lindsay. Reproducible measurement of single-molecule conductivity. Science, 294:571-574, 2001.

[9] Jeffrey M. Mativetsky, Giuseppina Pace, Mark Elbing, Maria A. Rampi, Marcel Mayor, and Paolo Samori. Azobenzenes as light-controlled molecular electronic switches in nanoscale metal-molecule-metal junctions. J. Am. Chem. Soc., 130:9192-9193, 2008. 
[10] G. Rubio-Bollinger, S. R. Bahn, N. Agraït, K. W. Jacobsen, and S. Vieira. Mechanical properties and formation mechanisms of a wire of single gold atoms. Phys. Rev. Lett., 87:026101, 2001.

[11] Bingqian Xu, Xiaoyin Xiao, and Nongjian J. Tao. Measurements of single-molecule electromechanical properties. J. Am. Chem. Soc., 125:16164-16165, 2003.

[12] Michael Frei, Sriharsha V. Aradhya, Max Koentopp, Mark S. Hybertsen, and L. Venkataraman. Mechanics and chemistry: single molecule bond rupture forces correlate with molecular backbone structure. Nano Lett., 11:1518-1523, 2011.

[13] Ilya V Pobelov, Gábor Mészáros, Koji Yoshida, Artem Mishchenko, Murat Gulcur, Martin R Bryce, and Thomas Wandlowski. An approach to measure electromechanical properties of atomic and molecular junctions. J. Phys.: Condens. Matter, 24:164210, 2012.

[14] Julie V. Macpherson, Patrick R. Unwin, Andrew C. Hillier, and Allen J. Bard. Insitu imaging of ionic crystal dissolution using an integrated electrochemical/AFM probe. J. Am. Chem. Soc., 118:6445-6452, 1996.

[15] Julie V. Macpherson and Patrick R. Unwin. Combined scanning electrochemicalatomic force microscopy. Anal. Chem., 72:276, 2000.

[16] Jeremy Abbou, Christophe Demaille, Michel Druet, and Jacques Moiroux. Fabrication of submicrometer-sized gold electrodes of controlled geometry for scanning electrochemical-atomic force microscopy. Anal. Chem., 74:6355-6363, 2002.

[17] Raul D.Rodriguez, Agnes Anne, Edmond Cambril, and Christophe Demaille. Optimized hand-fabricated AFM probes for simultaneous topographical and electrochemical tapping mode imaging. Ultramicroscopy, 111:973-981, 2011.

[18] Christine Kranz, Gernot Friedbacher, Boris Mizaikoff, Alois Lugstein, Jürgen Smoliner, and Emmerich Bertagnolli. Integrating an ultramicroelectrode in an AFM cantilever: combined technology for enhanced information. Anal. Chem., 73:2491-2500, 2001.

[19] A. Lugstein, E. Bertagnolli, C. Kranz, A. Kueng, and B. Mizaikoff. Integrating micro- and nanoelectrodes into atomic force microscopy cantilevers using focused ion beam techniques. Appl. Phys. Lett., 81:349-351, 2002.

[20] Angelika Kueng, Christine Kranz, Alois Lugstein, Emmerich Bertagnolli, and Boris Mizaikoff. Integrated AFM-SECM in tapping mode: simultaneous topographical and electrochemical imaging of enzyme activity. Angew. Chem. Int. Ed., 42:32383240, 2003.

[21] Justyna Wiedemair, Balamurali Balu, Jong-Seok Moon, Dennis W. Hess, Boris Mizaikoff, and Christine Kranz. Plasma-deposited fluorocarbon films: Insulation material for microelectrodes and combined atomic force microscopy-scanning electrochemical microscopy probes. Anal. Chem., 80:5260-5265, 2008. 
[22] Alois Lugstein, Emmerich Bertagnolli, Christine Kranz, and Boris Mizaikoff. Fabrication of a ring nanoelectrode in an AFM tip: novel approach towards simultaneous electrochemical and topographical imaging. Surf. Interface Anal., $33: 146,2002$.

[23] Heungjoo Shin, Peter J. Hesketh, Boris Mizaikoff, and Christine Kranz. Batch fabrication of atomic force microscopy probes with recessed integrated ring microelectrodes at a wafer level. Anal. Chem, 79:4769-4777, 2007.

[24] Waldemar Smirnov, Armin Kriele, René Hoffmann, Eugenio Sillero, Jakob Hees, Oliver A. Williams, Nianjun Yang, Christine Kranz, and Christoph E. Nebel. Diamond-modified AFM probes: From diamond nanowires to atomic force microscopy-integrated boron-doped diamond electrodes. Anal. Chem., 83:49364941, 2011.

[25] Yoshiki Hirata, Soichi Yabuki, and Fumio Mizutani. Application of integrated SECM ultra-micro-electrode and AFM force probe to biosensor surfaces. Bioelectrochem., 63:217-224, 2004.

[26] Terunobu Akiyama, Maurizio R. Gullo, Nicolaas F. de Rooij, Andreas Tonin, Hans-Rudolf Hidber, Patrick L. T. M. Frederix, Andreas Engel, and Urs Staufer. Development of insulated conductive probes with platinum silicide tips for atomic force microscopy in cell biology. Jpn. J. Appl. Phys., 43:3865-3867, 2004.

[27] Maurizio R. Gullo, Patrick L. T. M. Frederix, Terunobu Akiyama, Andreas Engel, Nico F. de Rooij, and Urs Staufer. Characterization of microfabricated probes for combined atomic force and high-resolution scanning electrochemical microscopy. Anal. Chem., 78:5436-5442, 2006.

[28] Patrick L T M Frederix, Maurizio R Gullo, Terunobu Akiyama, Andreas Tonin, Nicolaas F de Rooij, Urs Staufer, and Andreas Engel. Assessment of insulated conductive cantilevers for biology and electrochemistry. Nanotechnology, 16:9971005, 2005.

[29] Phillip S. Dobson, John M. R. Weaver, Mark N. Holder, Patrick R. Unwin, and Julie V. Macpherson. Characterization of batch-microfabricated scanning electrochemical-atomic force microscopy probes. Anal. Chem., 77:424-434, 2005.

[30] Andrew J. Wain, David Cox, Shengqi Zhou, and Alan Turnbull. High-aspect ratio needle probes for combined scanning electrochemical microscopy-atomic force microscopy. Electrochem. Commun., 13:78-81, 2011.

[31] Maksymilian A. Derylo, Kirstin C. Morton, and Lane A. Baker. Parylene insulated probes for scanning electrochemical-atomic force microscopy. Langmuir, 27:1392513930, 2011.

[32] A Avdic, A Lugstein, M Wu, B Gollas, I Pobelov, T Wandlowski, K Leonhardt, G Denuault, and E Bertagnolli. Fabrication of cone-shaped boron doped diamond and gold nanoelectrodes for AFM-SECM. Nanotechnology, 22:145306, 2011. 
[33] Kelly Leonhardt, Amra Avdic, Alois Lugstein, Ilya Pobelov, Thomas Wandlowski, Ming Wu, Bernhard Gollas, and Guy Denuault. Atomic force microscopy-scanning electrochemical microscopy: influence of tip geometry and insulation defects on diffusion controlled currents at conical electrodes. Anal. Chem., 83:2971-2977, 2011.

[34] Kelly Leonhardt, Amra Avdic, Alois Lugstein, Ilya Pobelov, Thomas Wandlowski, Bernhard Gollas, and Guy Denuault. Scanning electrochemical microscopy: Diffusion controlled approach curves for conical AFM-SECM tips. Electrochem. Commun., 27:29-33, 2013.

[35] Ole Bethge, Stephan Abermann, Christoph Henkel, and Emmerich Bertagnolli. Low temperature atomic layer deposition of high-k dielectric stacks for scaled metaloxide-semiconductor devices. Thin Solid Films, 517:5543-5547, 2009.

[36] N Chekurov, K Grigoras, A Peltonen, S Franssila, and I Tittonen. The fabrication of silicon nanostructures by local gallium implantation and cryogenic deep reactive ion etching. Nanotechnology, 20:065307, 2009.

[37] B. Schmidt, S. Oswald, and L. Bischoff. Etch rate retardation of $\mathrm{Ga}^{+}$-ion beamirradiated silicon. J. Electrochem. Soc., 152:G875-G879, 2005.

[38] Gábor Mészáros, Chen Li, Ilya Pobelov, and Thomas Wandlowski. Current measurements in a wide dynamic range-applications in electrochemical nanotechnology. Nanotechnology, 18:424004, 2007.

[39] Wujian Miao, Zhifeng Ding, and Allen J. Bard. Solution viscosity effects on the heterogeneous electron transfer kinetics of ferrocenemethanol in dimethyl sulfoxidewater mixtures. J. Phys. Chem. B, 106:1392-1398, 2002.

[40] Ilya V. Pobelov, Chen Li, and Thomas Wandlowski. Electrochemical scanning tunneling microscopy. In Bharat Bhushan, editor, Encyclopedia of Nanotechnology, pages 688-702. Springer, 2012.

[41] Michael V. Mirkin, Fu-Ren F. Fan, and Allen J. Bard. Direct electrochemical measurements inside a 2000 angstrom thick polymer film by scanning electrochemical microscopy. Science, 257:364-366, 1992.

[42] Gábor Nagy and Thomas Wandlowski. Double layer properties of $\mathrm{Au}(111) / \mathrm{H}_{2} \mathrm{SO}_{4}$ $(\mathrm{Cl})+\mathrm{Cu}^{2+}$ from distance tunneling spectroscopy. Langmuir, 19:10271-10280, 2003.

[43] Martin Hugelmann and Werner Schindler. In situ distance tunneling spectroscopy at $\mathrm{Au}(111) / 0.02 \mathrm{M} \mathrm{HClO}_{4}$ : from faradaic regime to quantized conductance channels. J. Electrochem. Soc., 151:E97-E101, 2004. 